\title{
La reproduction du lapin Angora de souche française : ovulation chez la femelle, production de semence chez le mâle
}

\author{
M Theau-Clément 1, RG Thébault 2, G Bolet 1 , $H$ de Rochambeau 1 \\ 1 INRA, station d'amélioration génétique des animaux, BP 27, 31326 Castanet-Tolosan Cedex; \\ 2 INRA, domaine pluridisciplinaire du Magneraud, BP 52, 17700 Surgères, France
}

(Reçu le 20 juin 1990; accepté le 2 septembre 1991)

\begin{abstract}
Résumé - Afin de mieux comprendre les problèmes de reproduction rencontrés chez les lapins Angora de souche française, nous avons étudié la fréquence et le niveau d'ovulation de 40 femelles, ainsi que l'ardeur sexuelle et la qualité de la semence de 8 mâles de cette souche. Cette expérience a été conduite en automne 1987 et renouvelée en hiver. Femelles : les lapines de 3 lots ont reçu respectivement $25,50 \mathrm{UI}$ de hCG, $0,8 \mu \mathrm{g}$ de $\mathrm{GnRH}$, les lapines du $4^{\theta}$ lot (témoin) ont été saillies. Les traitements hormonaux améliorent significativement la fréquence des lapines qui ovulent (respectivement $95,90,74$ et $28 \% ; P<0,05)$ mais sont sans influence sur le taux d'ovulation $(10,9 \pm$ 0,$7 ; 10,7 \pm 0,7 ; 11,3 \pm 0,8 ; 8,9 \pm 1,3$ corps jaunes par femelle ayant ovulé; $m \pm$ écart type de la moyenne, différences non significatives). Mâles : 2 éjaculats ont été prélevés 2 fois par semaine pendant 3,5 semaines, chez 8 lapins. Les ejaculats obtenus en mars sont significativement meilleurs que ceux récoltés en novembre pour le volume $(0,33$ vs $0,23 \mathrm{cc}, P<0,01)$, la motilité d'ensemble $(5,2$ vs $3,9, P<0,01)$, la motilité individuelle $(2,7$ vs $2,1, P<0,05)$, le nombre moyen de spermatozoïdes vivants par éjaculat $\left(71 \times 10^{6}\right.$ vs $\left.28 \times 10^{6}, P<0,01\right)$. Ces résultats permettent d'envisager une amélioration des performances de reproduction des lapins Angora de souche française par l'utilisation de l'insémination artificielle.
\end{abstract}

lapln Angora / ovulation / qualité de la semence

Summary - Reproduction of French Angora rabbits: ovulation rate of does and semen production of bucks. Ovulation rate and semen quality in French Angora rabbits were investigated to determine the potential of improving breeding practices in this breed. The proportion of does ovulating and their ovulation rate were studied in 40 females, as well as sexual behaviour and semen quality in 8 males. The experiments took place in autumn 1987 and were repeated in winter. Three groups of does were injected with 25, $50 \mathrm{IU}$ hCG or $0.8 \mu \mathrm{g}$ GnRH (groups 1,2 and 3, respectively). Another group was mated and served as a control (group 4). Hormonal treatments improved the proportion of does which ovulated $(95,90,74$ and $28 \%$ in groups $1-4$, respectively; $P<0.05)$ but did not change their ovulation rate $(10.9 \pm 0.7,10.7 \pm 0.7,11.3 \pm 0.8$ and $8.9 \pm 1.3$ corpora lutea per ovulating female, groups 1-4, respectively; $\mathrm{m} \pm \mathrm{SEM}, \mathrm{NS})$. In males, two ejaculates were collected twice weekly for 3.5 weeks from 8 bucks. Ejaculates obtained in March were better than those collected in November (volume: 0.33 vs $0.23 \mathrm{ml}, \mathrm{P}<0.01$; raw motility: 5.2 vs $3.9, P<0.01$; individual motility: 2.7 vs $2.1, \mathrm{P}<0.05$; number of living spermatozoa per ejaculate: 71 vs $28 \times 10^{6}, \mathrm{P}<0.01$, respectively). These results suggest that artificial insemination may be utilized to improve reproductive performance in French Angora rabbits. 


\section{INTRODUCTION}

Deux souches sélectionnées de lapins Angora sont exploitées dans le monde pour la production de poil : la souche française et la souche allemande. Les objectifs prioritaires de sélection ont été d'améliorer la productivité individuelle des sujets et la qualité du poil angora produit, sans accorder d'importance aux caractères de reproduction des animaux. Toutefois, avec l'amélioration des caractères de production poilière, les éleveurs semblent confrontés à des difficultés de reproduction : taux de gestation de $25 \%$ par saillie et tailles de portées de 4,9 lapereaux à la naissance (Thébault et Rochambeau, 1988). L'objet de cette étude est de mieux appréhender l'origine de ces problèmes en étudiant l'aptitude de la femelle à ovuler et celle du mâle à produire du sperme de bonne qualité.

\section{MATÉRIEL ET MÉTHODES}

\section{Potentialités ovulatoires des lapines}

Quarante lapines Angora de souche française, âgées de 5 à 53 mois, plage effective d'utilisation des femelles Angora dans les élevages français (Rochambeau et al, 1988) ont été utilisées. Trente-deux étaient nullipares, 1 primipare et 7 multipares.

Elles ont été élevées dans des cases individuelles en ciment, sur litière de paille avec eau de boisson à volonté. L'alimentation se composait de granulés concentrés complets (matières protéiques brutes $17 \%$, cellulose $17 \%$, matières minérales $10 \%$, acides aminés soufrés $0,75 \%$ ): $220 \mathrm{~g} / \mathrm{j}$ durant le mois suivant la récolte du poil, $180 \mathrm{~g} / \mathrm{j}$ le deuxième mois et $160 \mathrm{~g} / \mathrm{j}$ le troisième mois. Un jour de jeûne a été imposé chaque semaine et environ $400 \mathrm{~g}$ de paille de blé distribués la veille du jour de jeûne.

Quatre lots de 10 animaux ont été constitués. Les femelles du lot témoin ont été saillies.
L'ovulation des autres lapines a été provoquée par l'injection, selon les lots, de 25 ou $50 \mathrm{UI}$ de gonadotrophine chorionique (hCG Organon) ou de $0,8 \mu \mathrm{g}$ d'un analogue de GnRH (Réceptal Distrivet).

Chaque lot de femelles a été équilibré en fonction de l'âge, la parité et l'intervalle depuis la dernière récolte de poil. Le pourcentage de femelles ayant ovulé et le nombre moyen de corps jaunes ont été déterminés par examen endoscopique des ovaires $7 \mathrm{j}$ après le traitement selon la technique décrite par Theau-Clément et Bolet (1987). Une expérience préliminaire a montré que $93 \%$ des numérations sont exactes à 1 corps jaune près (données non publiées). L'essai a été réalisé en octobre 1987 et en février 1988 sur les mêmes femelles, sans qu'aucune lapine ne reçoive le même traitement dans les 2 séries.

Le pourcentage de lapines ayant ovulé a été analysé par le test de $\chi^{2}$ en appliquant la correction de continuité de Yates. Le nombre de corps jaunes par femelle ayant ovulé a été étudié avec un modèle d'analyse de variance incluant les effets période, traitement et leur interaction.

\section{Ardeur sexuelle et qualité de la semence des mâles}

Huit mâles âgés de 7 à 27 mois ont été utilisés pendant 3,5 semaines en novembre 1987 puis en mars 1988. Leurs conditions d'élevage ont été identiques à celles des femelles. La semence a été prélevée 2 fois par semaine (mardi et vendredi) à l'aide d'un vagin artificiel. Chaque fois, 2 éjaculats étaient récoltés à 10 min d'intervalle. L'ardeur sexuelle était mesurée par le temps écoulé entre l'introduction de la femelle dans la cage du mâle et l'éjaculation. L'attente d'une récolte n'a jamais dépassé $10 \mathrm{~min}$. Les méthodes d'évaluation qualitatives et quantitatives de la semence ont été décrites par Boussit (1989). Deux personnes ont réalisé les prélèvements selon la même méthode et la même séquence d'intervention : chacune un jour donné a prélevé tous les mâles. Les résultats ont été étudiés avec un modèle d'analyse de variance à effets fixés incluant les effets mâle, série, préleveur, numéro d'éjaculat et leurs interactions. 


\section{RÉSULTATS}

\section{Potentialités ovulatoires des lapines}

\section{Effet traitement}

Sur l'ensemble des 2 périodes expérimentales, le pourcentage des femelles qui ovulent ne varie pas significativement entre les 3 lots traités, mais pour chacun d'eux il est significativement plus élevé que celui du lot témoin (tableau I). Le traitement hormonal améliore donc significativement la fréquence d'ovulation des lapines (86\% contre $28 \%$ ).

Le nombre moyen de corps jaunes observés chez les femelles ayant ovulé varie de $8,9 \pm 1,3$ pour le lot témoin à $11,3 \pm 0,8$ pour le lot ayant reçu $0,8 \mu \mathrm{g}$ de $\mathrm{GnRH}$, sans différence significative; en particulier, $50 \mathrm{UI} \mathrm{hCG} \mathrm{ne} \mathrm{sont} \mathrm{pas} \mathrm{plus} \mathrm{efficaces} \mathrm{que}$ 25 UI $(10,7 \pm 0,7$ vs 10,9 $\pm 0,7)$.

\section{Effet période}

Il n'y a pas d'effet significatif de la période de traitement sur le pourcentage de lapines ovulantes et leur taux d'ovulation. Toutefois, on remarque une tendance à des résultats légèrement plus élevés en février qu'en octobre (respectivement +6 points, $+1,3$ corps jaune).

\section{Ardeur sexuelle et qualité de la semence des mâles Angora}

\section{Effet de la période}

Le volume, la motilité (d'ensemble ou individuelle) et le pourcentage de spermatozoïdes vivants sont significativement plus élevés en mars qu'en novembre (tableau II). II en résulte que les éjaculats récoltés en mars contiennent 2,5 fois plus de spermatozoïdes vivants que ceux récoltés en novembre $(P<0,01)$. Par contre, ni l'ar-

Tableau I. Effet du mode d'induction de l'ovulation sur la réponse des lapines.

\begin{tabular}{|c|c|c|c|c|c|c|}
\hline \multirow[t]{2}{*}{ Traitement } & \multicolumn{2}{|c|}{ Effectif } & \multicolumn{4}{|c|}{ Pourcentage des femelles ayant ovulé } \\
\hline & Octobre & Février & Octobre & & Février & Total \\
\hline 25 UI hCG & 9 & 10 & $89^{a}$ & & $100^{a}$ & $95^{a}$ \\
\hline 50 UI hCG & 9 & 10 & $89^{a}$ & & $90^{\mathrm{a}}$ & $90^{\mathrm{a}}$ \\
\hline $0,8 \mu \mathrm{g} \mathrm{GnRH}$ & 9 & 10 & $67^{a}$ & & $80^{\mathrm{a}}$ & $74^{a}$ \\
\hline $\begin{array}{l}\text { Saillie naturelle } \\
\text { (témoin) }\end{array}$ & 8 & 10 & $25^{b}$ & & $30^{\mathrm{b}}$ & $28^{\mathrm{b}}$ \\
\hline Total & 35 & 40 & 69 & NS & 75 & 72 \\
\hline
\end{tabular}

Résultats des tests $\chi^{2}$ : NS, non significatif; dans chaque colonne, les chiffres affectés de lettres différentes sont significativement differents $(P<0,05)$. 


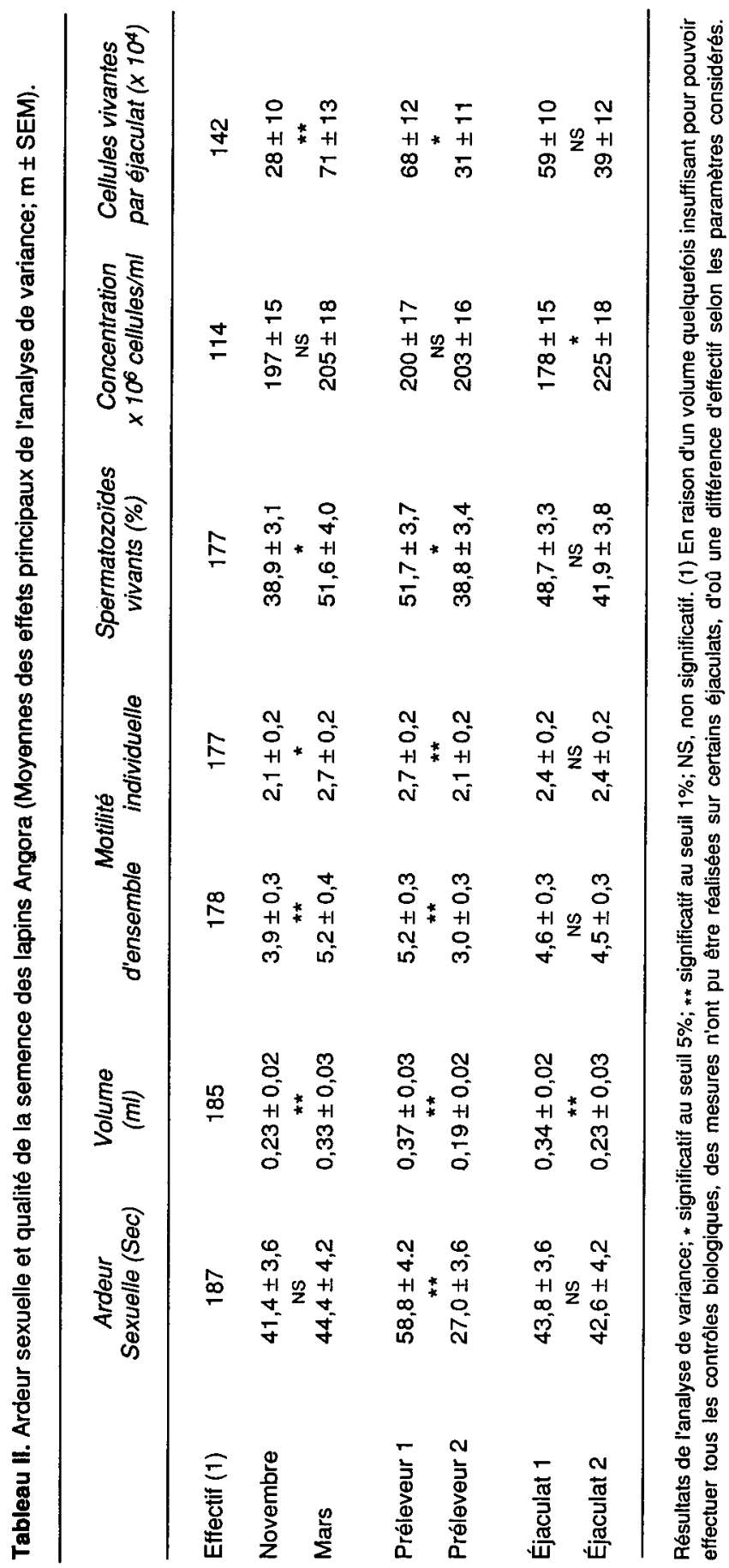


deur sexuelle des mâles ni la concentration des éjaculats ne varient significativement selon la période de récolte. II existe une interaction significative mâle $x$ période pour toutes les variables étudiées sauf l'ardeur sexuelle.

\section{Effet du numéro d'éjaculat}

Le volume du 1 er éjaculat est significativement plus élevé (+ $48 \%, P<0.01$ ) que celui du second. Le résultat est inverse pour la concentration en spermatozoïdes (+ $26 \%$ pour le $2^{2}$ éjaculat, $P<0,05$ ). II n'y a de différence significative pour aucun des autres paramètres (tableau II).

\section{Effet du préleveur}

Le préleveur 2 obtient tous les prélèvements imposés par le rythme choisi, contre $71 \%$ seulement pour le préleveur 1 . Le temps de récolte du préleveur 2 est significativement plus court que celui du préleveur $1(-54 \%, P<0.01)$. Par contre, les caractéristiques des éjaculats, à l'exception de la concentration, sont significativement plus élevées pour le préleveur 1 (tableau II). II existe une interaction préleveur $x$ éjaculat significative pour le volume, le pourcentage de spermatozoïdes vivants, la concentration et le nombre de cellules vivantes par éjaculat (tableau III).

\section{DISCUSSION}

\section{Potentialités ovulatoires des lapines Angora}

Cet essai met en évidence, chez les lapines Angora, des défauts d'ovulation importants en saillie naturelle. Les traitements hormonaux améliorent significativement la proportion des lapines qui ovulent mais pas leur nombre de corps jaunes. $\mathrm{Ce}$ dernier est du même ordre de grandeur que celui observé chez des lapines de chair (Bolet et al, 1988). Cette expérience ne révèle pas d'avantage significatif de l'un des traitements hormonaux par rapport aux autres. Toutefois, il faut rappeler que les gonadotrophines chorioniques, déjà utilisées pour l'étude de l'ovulation (Hulot et al, 1988) ont l'inconvénient d'entraîner rapidement la formation d'anticorps (Adams, 1972). Les facteurs hypothalamiques ne présentent pas ce handicap, comme l'ont démontré Schlolaut et al (1981) lors de traitements répétés. Cependant, il serait utile de déterminer la dose qui donne le meilleur taux d'ovulation chez la lapine Angora.

Chez les lapines de chair plus de $94 \%$ des femelles réceptives au mâle ovulent après une saillie naturelle ou à la suite d'une injection de GnRH (Theau-Clément

Tableau III. Interactions préleveur et numéro d'éjaculat sur les contrôles biologiques de la semence.

\begin{tabular}{ccccccc}
\hline Préleveur & $\begin{array}{r}\text { Numéro } \\
\text { d'éjaculat }\end{array}$ & $\begin{array}{c}\text { Volume } \\
(c c)\end{array}$ & Motilité & $\begin{array}{c}\text { Spermatozoödes } \\
\text { vivants } \%)\end{array}$ & $\begin{array}{c}\text { Concentration } \\
\left(\times 10^{6} \text { cellules }\right. \\
\text { par ml) }\end{array}$ & $\begin{array}{c}\text { Spermatozoïdes } \\
\text { vivants par } \\
\text { ejaculat }\left(x 10^{6}\right)\end{array}$ \\
\hline 1 & 1 & $0,47 \mathrm{a}$ & $5,5 \mathrm{a}$ & $60 \mathrm{a}$ & $232 \mathrm{a}$ & $101 \mathrm{a}$ \\
1 & 2 & $0,28 \mathrm{~b}$ & $4,9 \mathrm{ab}$ & $43 \mathrm{ab}$ & $166 \mathrm{~b}$ & $34 \mathrm{~b}$ \\
2 & 1 & $0,21 \mathrm{~b}$ & $3,6 \mathrm{~b}$ & $37 \mathrm{~b}$ & $122 \mathrm{~b}$ & $17 \mathrm{~b}$ \\
2 & 2 & $0,18 \mathrm{~b}$ & $4,1 \mathrm{~b}$ & $41 \mathrm{~b}$ & $283 \mathrm{a}$ & $44 \mathrm{~b}$ \\
\hline
\end{tabular}

Dans chaque colonne, les chiffres affectés de lettres différentes sont significativement différents $(P<0,05)$. 
et al, 1990). Toutes les femelles Angora saillies dans le présent travail étaient réceptives, leurs défauts d'ovulation restent donc inexpliqués. II est cependant peu probable qu'ils soient liés à un manque de follicules mûrs dans l'ovaire, puisque après traitement hormonal (GnRH ou hCG), les taux d'ovulation observés sont normaux. Le problème se pose donc en amont de l'hypophyse.

Ces résultats semblent donc indiquer que l'on peut lever partiellement les problèmes de reproduction des lapines Angora de souche française en induisant l'ovulation par un traitement hormonal. Toutefois, la confrontation des taux d'ovulation observés dans cet essai avec une prolificité de 4,9 lapereaux par portée enregistrée dans cet élevage par Thébault et Rochambeau (1988) suggère un problème supplémentaire de mortalité embryonnaire.

\section{Ardeur sexuelle et qualité de la semence des mâles Angora}

Les résultats observés sur les éjaculats récoltés en mars sont significativement supérieurs à ceux des éjaculats récoltés en novembre, confirmant l'effet de la période de récolte déjà signalé chez des mâles Angora de souche allemande ( $\mathrm{Hu}$ et al, 1988). II est toutefois prématuré de conclure à un effet lié à la saison.

Pour chacun des paramètres élémentaires mesurés, nous observons des différences significatives entre mâles. Par contre le critère global que représente le nombre de spermatozoïdes vivants par éjaculat, ne diffère pas significativement entre mâles. Les valeurs prises par ce critère semblent d'un bon niveau, si on les compare à celles des lapins de chair. La semence de cet échantillon de mâles Angora de souche française ne présente donc pas de défauts majeurs. II conviendra cependant de confirmer cette observation en étudiant le pouvoir fécondant.

Ce travail souligne aussi l'importance des conditions de prélèvements. Une interaction significative est observée entre le préleveur et le numéro d'éjaculat pour les différentes caractéristiques de ce dernier. Le tableau III montre la supériorité, du 1 er éjaculat obtenu par le préleveur 1 qui, moins expérimenté, mettait 2 fois plus de temps à récolter les mâles, mais récoltait 2 fois plus de spermatozoïdes vivants par éjaculat que le préleveur 2. La prise en compte des seuls cas où les 2 prélèvements ont eu lieu ne modifie pas ces conclusions. Ceci confirme que la préparation de la récolte a une influence sur la quantité et la qualité de la semence obtenue et qu'elle ne doit pas être trop rapide (McMillan et Hafs, 1967).

En conclusion, ce travail permet d'envisager le recours à l'insémination artificielle pour la reproduction des lapins Angora puisqu'une bonne induction de l'ovulation est obtenue par un traitement hormonal des femelles et que des conditions de récolte appropriées des mâles fournissent un nombre élevé de spermatozoïdes. II faudra vérifier si la fertilité et la prolificité qui résultent de ces pratiques sont également améliorées.

\section{REMERCIEMENTS}

Les auteurs remercient $M$ Bonnet, $G$ Blanié, $J$ Esparbié et J Lanta pour leur contribution technique à cette étude.

\section{RÉFÉRENCES}

Adams CE (1972) Induction of ovulation and artificial insemination technics in the rabbit. Vet Rec $91,194-197$ 
Bolet G, Brun JM, Hulot F (1988) Relationships between ovulation rate and embryonic survival in various strains of rabbits. In: 4th Congr World Rabbit Sci Assoc (S Holdas, ed), 1014 oct 1988, Budapest, 2, 149-157

Boussit D (1989) Reproduction et insémination artificielle en cuniculture. Assoc Fr Cuniculture, Lempdes France, $234 p$

Hu JF, Hong ZY, Leng HR, Wang QX (1988) The variabilities in the semen quality of semen of German angora and China angora in summer and autumn. In: 4th Congr World Rabbit Sci Assoc (S Holdas, ed), 10-14 oct 1988, Budapest, 2, 524

Hulot F, Mariana JC, Cattiau G (1988) hCGinduced ovulation in two rabbit breeds: effects of dose, season and sexual behaviour. Livest Prod Sci 20, 257-267

McMillan KL, Hafs HD (1967) Semen output of rabbit ejaculated after varying sexual preparation. Proc Soc Exp Biol Med 125, 1278 1281

Rochambeau $H$ de, Thébault RG, Loyer $G$ (1988) Some aspects of the demographic structure of the French angora rabbit breed.
In: 4th Congr World Rabbit Sci Assoc (S Holdas, ed), Budapest, 10-14 octobre 1988, 2, 218-226

Schlolaut W, Lange K, Paufler S (1981) Einfluss des Thioaminosäurengehaltes im Futter, des Alters und der Geburtsauslösung mit Oxytocin auf die Reproduktionsleistung des Kaninchens bei post-partaler Insemination. Züchtungskunde 53, 283-294

Theau-Clément M, Bolet G (1987) Mesure du taux d'ovulation par coelioscopie chez la lapine. Reprod Nutr Dév 27, 701-705

Theau-Clément M, Bolet G, Roustan A, Mercier $P(1990)$ Comparaison de différents modes d'induction de l'ovulation chez les lapines multipares en relation avec leur stade physiologique et la réceptivité au moment de la mise à la reproduction. In: $5^{\circ}$ Journ Rech Cunicole. INRA-ITAVI Paris, 12-13 décembre 1990,1 , communication $n^{\circ} 6$

Thébaut RG, Rochambeau $H$ de (1988) Population data and demographic parameters of a French angora rabbit strain. In: 4th Congr World Rabbit Sci Assoc (S Holdas, ed), Budapest, 10-14 octobre 1988, 2, 227-238 